\title{
Patients' perceptions of a pressure ulcer prevention care bundle in hospital: a qualitative descriptive study to guide evidence-based practice
}

Short title: Patient perceptions of a pressure ulcer prevention care bundle

\section{$\underline{\text { Authors }}$}

Shelley Roberts (PhD), Research Fellow, Griffith University (corresponding author)

Address: NHMRC Centre of Research Excellence in Nursing, Menzies Health Institute Queensland, Griffith University, Gold Coast Campus, QLD 4222 Australia

Phone: +617 55529557

Fax: +617 55529144

Email: s.roberts@griffith.edu.au

Marianne Wallis (PhD), Professor of Nursing, University of the Sunshine Coast

Address: School of Nursing, Midwifery and Paramedicine, University of the Sunshine Coast, Locked Bag 4, Maroochydore DC, QLD 4558 Australia

Phone: +61754565032 ～Fax: +61754565940 $\quad$ Email: mwallis@usc.edu.au

Elizabeth McInnes (PhD), Deputy Director, Nursing Research Institute, St Vincent’s Health Australia (Sydney) and Australian Catholic University.

Address: School of Nursing, Midwifery and Paramedicine, Australian Catholic University, North Sydney NSW 2060 Australia

Phone: +612 83823790

Fax: (02) 83823792

Email: liz.mcinnes@acu.edu.au

Tracey Bucknall (PhD), Professor and Associate Head of School (Research), Centre for Quality and Patient Safety, School of Nursing and Midwifery, Deakin University; Foundational Chair in Nursing, Alfred Health.

Address: Centre for Quality and Patient Safety, School of Nursing and Midwifery, Deakin University, Geelong, VIC 3220 Australia

Phone: +61 $392446529 \quad$ Fax: $\quad$ Email: tracey.bucknall@deakin.edu.au

Merrilyn Banks (PhD), Director of Nutrition and Dietetics, Royal Brisbane and Women’s Hospital

Address: Department of Nutrition and Dietetics, Royal Brisbane and Women’s Hospital, Herston, QLD 4019

Australia

Phone: +617 36467994

Fax: +61736461874＜wide>Email: Merrilyn.Banks@health.qld.gov.au

Lauren Ball (PhD), Research Fellow, NHMRC Centre for Research Excellence in Nursing, Griffith University

Address: NHMRC Centre of Research Excellence in Nursing, Menzies Health Institute Queensland, Griffith University, Gold Coast Campus, QLD 4222 Australia

Phone: +617 55529702

Fax: +61755529144

Email: 1.ball@griffith.edu.au

Wendy Chaboyer (PhD), Director of NHMRC Centre for Research Excellence in Nursing, Griffith University

Address: NHMRC Centre of Research Excellence in Nursing, Menzies Health Institute Queensland, Griffith University, Gold Coast Campus, QLD 4222 Australia

Phone: +617 55529702

Fax: +61755529144

Email: w.chaboyer@griffith.edu.au 


\section{ABSTRACT}

Background: Pressure ulcers (PU) place a significant burden on patients and hospitals. Our team developed and tested a pressure ulcer prevention care bundle (PUPCB) in a cluster randomised trial. As part of the process evaluation conducted alongside the trial, we explored patients’ perceptions of the intervention.

Aims: To identify patients’ perceptions and experiences of a PUPCB in hospital.

Methods: This qualitative descriptive study explored the perceptions of a subset of patients who participated in a trial testing the PUPCB across four intervention hospitals. A trained interviewer conducted semi-structured interviews, which were digitally recorded, transcribed and analysed using thematic analysis.

Findings: Nineteen patients were interviewed across the four hospitals. Three main themes emerged: 1) Importance of personal contact in PUPCB delivery; 2) Understanding PUP enhances participation; and 3) Individual factors impact patients’ engagement in PUP.

Discussion: The extent to which patients adopted the intervention appeared to be influenced by the complexity of education materials, compatibility with patients' existing knowledge and beliefs, and perceived advantage of the intervention; ability for human interaction; and patient-related facilitators and barriers to participating in PUP care.

Implications for Practice: From the perspectives of patients, three simple messages can enable participation in PUP care. The extent to which patients and nurses partner in care, through exchange of information and mutual involvement in PUP activities, also appears to be an important aspect of participation in PUP. 
Linking Evidence to Action: This study found patients accepted a PUPCB that encouraged participation in care, particularly as it involved personal and positive interactions with nurses and provision of information that was easy to understand and resonated with patients.

Keywords: Care bundle; patient-centred care; patient participation; pressure injury prevention; pressure ulcer prevention. 


\section{BACKGROUND}

Pressure ulcers (PU) are a significant problem in the clinical setting. PUs cause severe physical, social and psychological problems for patients, including pain, reduced mobility and functional ability, restricted daily and social life activities, mood changes and emotional problems and poor self-image (Gorecki et al., 2009; Gorecki, Closs, Nixon, \& Briggs, 2011). For hospitals, PUs cause substantial economic burden through longer length of stay, high treatment costs (Graves \& Zheng, 2014; Nguyen, Chaboyer, \& Whitty, 2015) and financial penalties to institutions (Queensland Government \& Queensland Health, 2012). Pressure ulcer prevention (PUP) is therefore a national and international priority for health care organisations (Australian Commission on Safety and Quality in Health Care, 2011; European Pressure Ulcer Advisory Panel, National Pressure Ulcer Advisory Panel, \& Pan Pacific Pressure Injury Alliance, 2014).

Our team recently developed a pressure ulcer prevention care bundle (PUPCB) (citation blinded for peer review) and tested its effectiveness and cost-effectiveness in a cluster randomised trial (the INTroducing A Care bundle To prevent pressure injury (INTACT) trial) in eight Australian hospitals (citation blinded for peer review). The PUPCB promoted patient participation in PUP care and was delivered to both patients and nurses. Patients received PUP education with a brochure, poster and DVD, which contained three messages: 1) Keep moving; 2) Look after your skin; and 3) Eat a healthy diet. Whilst a significant reduction in hospital acquired PU was seen in intervention patients at the cluster level, the difference was not statistically significant at the individual patient level when adjusted for covariates and clustering. Despite a sample size of 1598 patients, the study was found to be underpowered, with a larger than expected intra-class correlation coefficient and a small number of clusters (eight). It is important to understand why, how and for whom the intervention may have 
worked (or not) in order to interpret the results of the main trial and consider implications for future research and practice.

A pre-specified process evaluation (i.e. planned prior to trial commencement) was conducted alongside the INTACT trial to understand the processes underpinning its implementation and how these may have affected study outcomes (Craig et al., 2008). The process evaluation was guided by a framework proposed for cluster randomised trials (Grant, Treweek, Dreischulte, Foy, \& Guthrie, 2013). The main evaluation domains in this framework include recruitment and reach, intervention delivery, and response to the intervention; for both individuals (patients) and clusters (hospitals). The framework also considers context, maintenance of the intervention, and findings of the main trial. The overall process evaluation for the INTACT trial is reported elsewhere (Chaboyer et al., 2016). This paper specifically reports in detail on individuals' (patients') response to the intervention. Nurses' perceptions of the bundle are reported elsewhere (Roberts et al., 2016). Understanding how patients perceive and respond to an intervention provides insight into its acceptability, effectiveness and likelihood of being sustained in the longer term. It is especially important to assess individuals' response to an intervention that requires adherence or behaviour change (Grant et al., 2013). This study aimed to explore patients’ perceptions of a PUPCB, including its acceptability and usefulness to patients and their perceived facilitators and barriers to participation in PUP care. These insights are important for interpreting the main trial findings and in considering future use of the PUPCB. 


\section{METHODS}

\section{Study design}

This qualitative descriptive study (Sandelowski, 2000) consisted of semi-structured interviews with a subset of patients participating in the INTACT trial at each of four intervention sites, to explore their perceptions of and response to the PUPCB. Ethical approval was gained at each intervention site and from the participating university for patient interviews. This study comprised the ‘individuals’ response to intervention’ domain of Grant et al.’s framework for process evaluations of cluster randomised trials (see Figure 1).
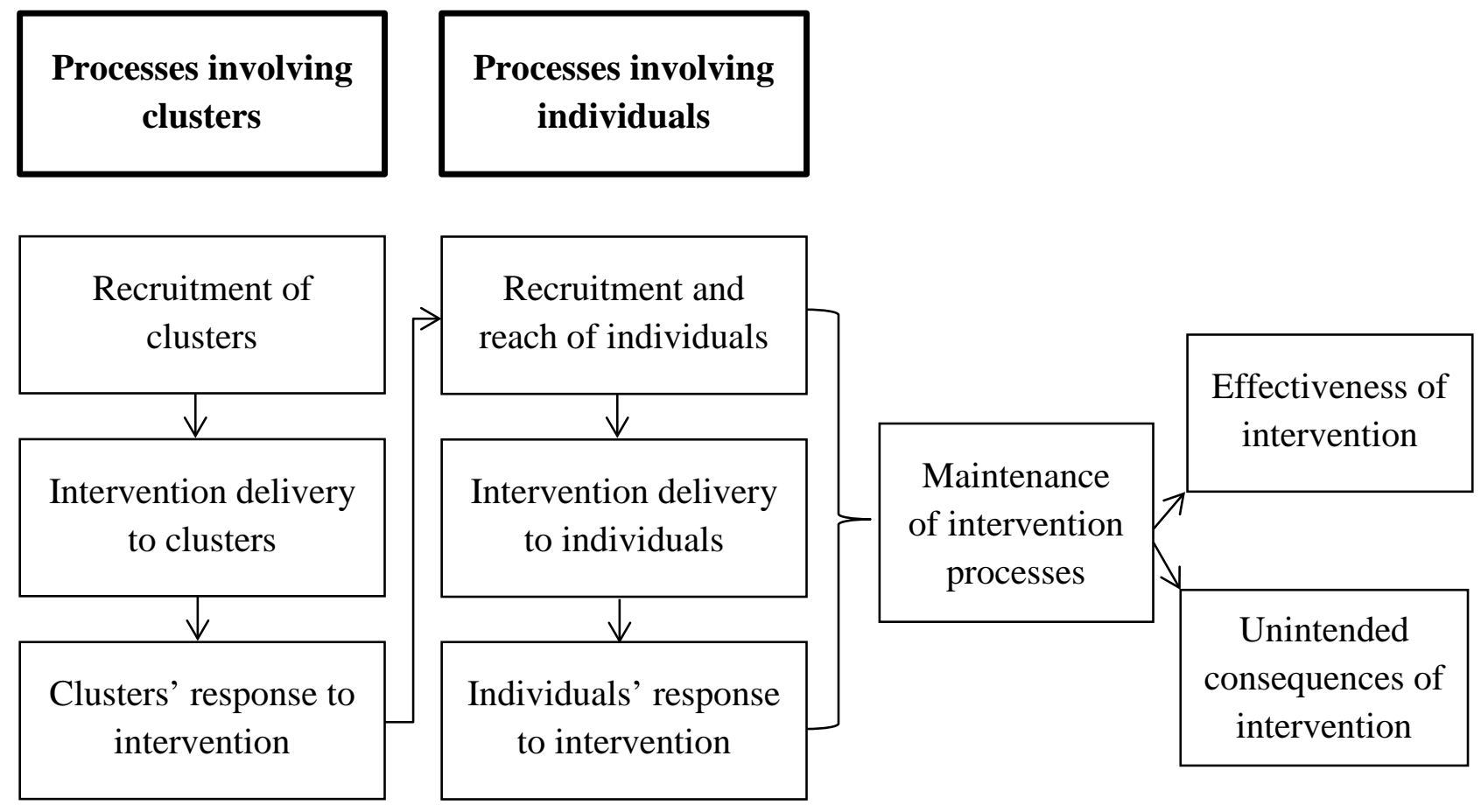

Theory underpinning intervention

\section{Context}

Figure 1: Framework for process evaluations of cluster randomised trials (adapted from Grant et al. (2013)) 


\section{Setting}

This study was conducted in four acute hospitals (consisting of both public and private hospitals, across two Australian states) that were intervention sites for the INTACT trial.

\section{Participants and recruitment}

Patients were eligible for inclusion if they had already consented to participate in the INTACT trial, were randomised to the intervention group, and agreed to an interview. Inclusion criteria for the main trial are reported elsewhere (Chaboyer et al., 2016). Purposive sampling was used to include a mix of male and female, older and younger, medical and surgical patients, with and without experience of PU. Each patient was provided with a participant information sheet outlining the study and informed consent was gained from agreeable patients.

\section{Data collection}

A semi-structured interview guide was developed based on previous literature (Latimer, Chaboyer, \& Gillespie, 2014; Whiting, 2008) and piloting of the intervention (Gillespie, Chaboyer, Sykes, O'Brien, \& Brandis, 2014). Questions were structured within three domains: (1) Remembering and understanding the intervention; (2) Using and participating in the intervention; and (3) Perceived value of the intervention. Example questions and prompts for each domain are shown in Table 1.

The interview guide was reviewed by several team members experienced in qualitative research and refined prior to the study. It was piloted in practice interviews (described in the next paragraph) and study site investigators provided feedback, which was used to further refine the guide. 
Table 1: Semi-structured interview domains and example questions

\begin{tabular}{|c|c|}
\hline Domain & Example questions and prompts \\
\hline $\begin{array}{l}\text { 1) Remembering } \\
\text { and } \\
\text { understanding the } \\
\text { intervention }\end{array}$ & $\begin{array}{l}\text { - What did you know about pressure ulcers and pressure ulcer } \\
\text { prevention before you came into hospital? } \\
\text { - Do you remember someone coming to speak to you about pressure } \\
\text { ulcers? What can you remember about this? What did they tell you? }\end{array}$ \\
\hline $\begin{array}{l}\text { 2) Using and } \\
\text { participating in } \\
\text { the intervention }\end{array}$ & $\begin{array}{l}\text { - Which parts of the study did you find the most interesting or } \\
\text { engaging? Can you give examples? } \\
\text { - When you think about your time in hospital, can you remember any } \\
\text { instances when you used the information that you were given about } \\
\text { pressure ulcers? For example, did you do anything differently? Why } \\
\text { or why not? }\end{array}$ \\
\hline $\begin{array}{l}\text { 3) Perceived } \\
\text { value of the } \\
\text { intervention }\end{array}$ & $\begin{array}{l}\text { - Do you think this pressure ulcer prevention information was of any } \\
\text { value to you personally? Why or why not? } \\
\text { - Will you use this information again in the future? How? }\end{array}$ \\
\hline
\end{tabular}

A training manual was developed for interviewers, containing information on study background, interviewing technique, preparation for data collection (including practice interviews), recruitment and informed consent, interview process and ongoing development of interviewer and interview guide. This training manual was used by the study investigator at each intervention site to train one interviewer for that site. After receiving this training, interviewers conducted at least one practice interview with a patient under the supervision of the study site investigator, who provided feedback and further training specific to that interviewer. Pilot interview data were not included in the analysis.

Data were collected between November 2014 and March 2015. Interviews were conducted at the patient's bedside or other private area at a time of convenience for patients. The semistructured interview guide was used to facilitate a conversational style of interviewing; their responses were used to guide the direction of the conversation and determine which prompts or questions were asked next. Interviews lasted approximately 15-20 minutes and were 
audiotaped and transcribed for analysis. Interviews continued until data saturation was reached (i.e. no new ideas or themes were identified).

\section{Data analysis}

Interview data were analysed using thematic analysis (Braun \& Clarke, 2006; Vaismoradi, Turunen, \& Bondas, 2013). One member of the team led data analysis and transcribed interviews to become familiar with the data. Two independent researchers read and reread transcripts, to allow them to become immersed in the data, and developed codes based on the verbatim statements of participants. Codes were compared and contrasted and aggregated into groups, which became sub-themes; and these were grouped into themes based on common threads throughout the codes. Development of labels for sub-themes and themes was an iterative process; they were discussed with the team, reviewed and revised accordingly. Researchers constantly referred back to the raw data to validate themes and sub-themes. Trustworthiness was achieved by employing several strategies. Firstly, frequent discussion among the research team ensured the codes accurately reflected the data and themes and subthemes adequately encompassed the data (i.e. credibility). Interviews were conducted within a short time frame at each site and interviews were transcribed concurrently, which started the analytic process (i.e. to maximise dependability). Finally, purposive sampling was used to ensure a broad representation of patients across all the study sites (i.e. transferability).

\section{FINDINGS}

A total of 19 patients participated in interviews across the four intervention sites. Three sites recruited five patients and one site recruited four patients. Ten patients (53\%) were female. The mean $( \pm \mathrm{SD})$ age of all patients was $68.8( \pm 16.5)$ years (range $31-96)$. At the time of the interview, their mean length of stay was 7.8 ( \pm 5.6$)$ days (range 3-24) and mean length of 
time since recruitment into INTACT was 6.9 ( \pm 5.8 ) days (range 2-23). Patients were recruited from medical $(n=5,26 \%)$ or surgical $(n=14,74 \%)$ wards.

Patients expressed a number of ways by which they did or did not participate in PUP care, by engaging in the intervention or encountering barriers to participation. Participants' perceptions of and experience with the intervention are described in three themes: 1) Importance of personal contact in PUPCB delivery; 2) Understanding PUP enhances participation; and 3) Individual factors impact patients' engagement in PUP.

\section{Importance of personal contact in PUPCB delivery}

Patients described a range of responses to the PUPCB. The importance of human contact in intervention delivery was often highlighted by patients and they remembered and understood the key messages to varying degrees. There were mixed responses to the resource materials (i.e. the brochure, poster and DVD), with some patients finding them useful and informative, and others finding them difficult to remember or unhelpful.

(a) Valuing human interaction

Most patients expressed that verbal communication and interaction with the trial's research assistants (RAs) was the most important, useful and valuable part of the intervention.

I thought the ladies coming in each day were more useful. (P12, Site 3)

Patients conveyed that the skin checks, assistance, encouragement and advice provided by the outcome assessor RAs were extremely useful and made them feel valued and important. Patients enjoyed daily visits from outcome assessor RAs and expressed the human presence acted as a reminder and motivator to enact PUP strategies. In particular, many patients 
highlighted the importance of having continuity or a constant in their care (i.e. the same person visiting each day).

It's very nice to have someone coming around; at least the interest is there, you know. It makes a lot of difference... Mentally as well as physically. (P1, Site 1)

I think by people coming in, it reminds you all the time. (P15, Site 3)

(b) Recalling and understanding the key messages

Patients mentioned the three key messages of the intervention: keep moving, eat a healthy diet and look after your skin. Most patients could recall either all three or at least one of the messages, whilst very few patients remembered none. Moving was the most commonly mentioned message, followed by nutrition and then skin care. Many patients said they remembered the initial discussion around PUP with the interventionist RA better than the other educational materials (i.e. brochure, poster, DVD). Most patients found the information useful, but not necessarily interesting for some.

To keep moving, to eat a healthy diet and to look after my skin. (P7, Site 2)

Three things but I can only remember the movement one... That's about all I can remember at the moment. (P17, Site 4)

(c) Variable acceptance of the PUPCB resource materials

Patients had mixed responses to the three PUPCB resource materials; the brochure, the poster and the DVD. As described in the sub-theme 'Importance of human interaction', many patients preferred verbally engaging with people to reading or watching the information. However, many patients still found the resources useful. Patients thought the brochure was 
informative, simple and concise and reported they would use it again in the future for themselves or for a loved one. However, a few patients found the brochure difficult to remember or felt they did not learn anything new from it. Others found it burdensome due to the large amount of paperwork they had already received in hospital.

The brochures were very informative. And that's something I can take with me and read over and over and watch out for signs of pressure sores. (P6, Site 2)

I think there was a pamphlet too. I've been given so much paperwork that it's hard to go through it. (P15, Site 3)

They keep on giving me bits of paper... I understood it... All these red dots - I know where to watch. (P18, Site 4)

The patients who remembered or could see the poster found it helpful to have the main points of the brochure summarised in a visually pleasing way. Patients liked being able to see it all the time and reported that it acted as a reminder of the PUPCB's key messages. Other patients however, could not remember seeing the poster, or reported it was placed too far away for them to read.

To have the reminder up there was good. (P11, Site 3)

Patients expressed varying opinions on the usefulness of the DVD. However, all those who remembered watching it found it uncomplicated and easy to understand. Some patients found it informative and felt it increased their awareness of PU and how they may be prevented. Patients liked that the DVD was short in duration as they found it difficult to concentrate in the hospital environment. Others found the DVD boring, or would have preferred something they could more actively engage in, such as a program on a computer or iPad. 
Maybe more interactive... Or maybe something on an iPad rather than a video. Because sometimes if you're just sitting there watching you're not really paying attention. (P4, Site 1)

The video was quite short, because quite often in hospital you're being poked and prodded left and right so you can only really do something for five minutes...just a few important messages without complicating the story. (P2, Site 1)

\section{Understanding PUP enhances participation in care}

This theme describes how patients responded positively to the intervention by actively engaging in PUP care. There seemed to be three ways by which the intervention encouraged patients to enact their own PUP strategies. Firstly, patients expressed how their natural instincts and previous knowledge were reinforced by the messages of the PUPCB, which had a positive impact on their participation. They also described how the knowledge gained from the PUPCB empowered them to enact PUP strategies. Finally, patients expressed that participating in the intervention increased their awareness of PU, which motivated them to actively carry out PUP strategies.

(a) Reinforcing routines and existing knowledge

Patients described how the main messages of the PUPCB reinforced what they instinctively did or what they already knew. Some explained that before receiving the PUPCB, they were not aware that their behaviours or practices were important for PUP. Many patients described how they already moved or repositioned themselves in bed as a natural reaction to feeling uncomfortable or to relieve pressure in certain areas. Patients also reported that using moisturisers for their skin and having a healthy diet were practises they already undertook as they were aware of their general importance, but had not always associated these with PUP. 
Patients expressed how these behaviours were reinforced by the information given in the PUPCB.

That makes sense - nutrition... You can't have good skin if you're not eating the proper foods. I guess it was something you know which has been reinforced. (P2, Site 1)

Having a prior knowledge of what to look out for, I think naturally if you felt in any areas that 'oh my tailbone, I shouldn't be sitting, I'd better lay down' - well it's only common sense isn't it? (P18, Site 4)

I do take care of my skin but (this) reinforces it. (P7, Site 2)

(b) Gaining knowledge facilitates participation

Patients expressed they were empowered to actively participate in and take ownership of PUP strategies through the knowledge gained from the PUPCB. Patients described how they enacted the key messages of the PUPCB around mobility, skin health and nutrition for their own PUP. Many patients felt that being given this information 'allowed' them to participate in their PUP care rather than relying on staff.

...be more vigilant with my skin, and checks. Just sort of not think 'oh well I'm in hospital, the nurses can do it'. (P14, Site 3)

I'm sure my (heat) rash would have gotten far worse if I wasn't given the advice I was given... I knew how to cope with it. Knowledge is always power, so any bit of extra knowledge is always good. (P2, Site 1)

(c) Awareness is motivating 
In this sub-theme, patients expressed how the intervention increased their awareness of the possibility of developing a PU, potential consequences and strategies for prevention. Many patients did not realise they could be at risk of PU during hospitalisation until being enrolled in the study. They described how participating in the intervention made them aware of their own individual risk factors and ways by which they could minimise this risk. Patients expressed the increased awareness of PU acted as a motivator to participate in PUP.

But since then, myself, I've been aware of it.... at night-time I try to change my positioning so that I'm not lying on my back... I'd lie on one side for half the night to give the centre of my back a bit of a break. So it all came from this. (P11, Site 3)

\section{Individual factors impact patients' engagement in PUP}

Patients discussed a number of reasons why they may not have been as actively involved in the PUPCB or in their PUP care. These included having a poor understanding of PUP through lack of experience, low perceived importance and failing to acknowledge own PU risk; and individual and care-related barriers to participation in PUP.

(a) Lack of understanding of PU

Patients expressed having limited personal experience with PU resulted in poor knowledge and a lack of understanding about the importance of PU and PUP strategies prior to receiving the PUPCB. Patients associated hospitalisation with being exposed to PUP education; patients who had never been hospitalised before described this as a reason for having limited PU knowledge. Other patients expressed surprise at never receiving PU education in previous hospitalisations. 
I have never had personal experience but I have heard of it.... I had known some people who did (have PU) but it didn't mean much to me because it wasn't me was it? (P15, Site 3)

Bed sores was nothing I'd had to think about before having more or less been healthy. (P2, Site 1)

Many patients failed to acknowledge their own risk of PU, despite being aware they were deemed 'at-risk' of PU and were included in the study. Patients described perceived risk factors of PU that did not relate to themselves or their current situation. They thought 'other' patients would be at risk, such as patients who were older, less mobile, or in hospital for longer; but did not consider themselves as having the potential to develop PU. Many patients reported being aware of PU but never considered it to be a problem they would encounter.

I'm still mobile... Honestly I don't think I'd get a bed sore. (P18, Site 4)

(b) Personal barriers to participating in PUP

Some patients described a lack of engagement with the PUPCB, which seemed to be associated with poor participation in PUP care. Commonly perceived barriers to participating in PUP included patient-related factors such as age, cognition and mobility. Patients explained how medications and recent surgery or anaesthesia resulted in tiredness and difficulty in concentrating on the education and resources provided in the PUPCB. A busy hospital environment also seemed to contribute to poor recollection of the PUPCB. This resulted in patients finding it difficult to recall the messages of the PUPCB due to the timing of its delivery. 
...You have to move, even when you lie in bed... If you sit down you have to move... Unfortunately... when you be a certain age, it's not so easy. You get tired. (P5, Site 1)

I don't remember (the messages) because... I hadn't long gotten over the anaesthetic that I'd had. (P15, Site 3)

...'Cause there's so much been going on... I'd forgot all about it. (P18, Site 4)

Some patients expected that PUP care was the responsibility of nursing staff and therefore took a passive approach to PUP. Others expressed this was particularly the case for patients who are unable to attend to their own PUP cares.

I thought the nurses were supposed to keep checking on you. (P11, Site 3)

I haven't had time to do anything, you know. I've just been laying there. (P1, Site 1)

\section{DISCUSSION}

This descriptive qualitative interview study assessed patients' response to a PUPCB for preventing hospital acquired PU in at-risk patients. Patients' perceptions of the intervention were represented in three themes: 1) Importance of personal contact in PUPCB delivery; 2) Understanding PUP enhances participation; and 3) Individual factors impact patients' engagement in PUP. In these themes, patients described facilitators and barriers to participating in the intervention and in PUP care in general, and provided feedback on intervention components and processes. These insights are essential to consider when interpreting results from the trial and planning implementation into practice. That is, the extent to which patients actively engaged in the PUPCB intervention likely influenced its effect. These findings are important to consider alongside nurses' perceptions of the 
intervention (reported elsewhere); which overall were positive and included insights into how the intervention may be sustained in practice (Roberts et al., 2016).

Overall, patients responded positively to the intervention, describing how they understood the key messages, responded to the PUPCB materials and valued human interaction. While most patients described some degree of participation in PUP cares, many factors seemed to influence this participation. In particular, patients’ knowledge and understanding appeared to affect participation; whether it was existing knowledge, gained knowledge or awareness. According to Rogers' 'Diffusion of Innovation' theory, several elements relating to aspects of the intervention itself and the nature of those using it may play a role in its adoption (Rogers, 2010). For example, the complexity, compatibility, relative advantage, trialability and observability of the intervention as perceived by those exposed to it, can determine its rate of adoption (Rogers, 2002). The extent to which the PUPCB was adopted by patients in the current study seemed to be influenced by many of these elements, which were evident in their responses as discussed below.

In this study, the majority of patients found the three key messages of the PUPCB informative, useful and uncomplicated. Rogers suggests that complexity of an intervention, that is the degree to which it is perceived as difficult to understand and use, influences its uptake (Rogers, 2002). In the current study, the resource materials were developed considering that hospitalised patients may have low health literacy (Australian Bureau of Statistics, 2008), and extensive piloting and feedback were integral in their development to enhance comprehensibility (Gillespie et al., 2014). Findings indicate barriers to patients understanding the intervention were not around its complexity; rather, it was difficult to remember in the context of being a hospital inpatient. The timing of intervention delivery was deemed crucial by patients; being drowsy, sedated, acutely unwell or post-surgical, or the 
busy hospital environment made it difficult for patients to concentrate or remember the education. Other patients mentioned the brochure was just adding to the many paper resources they already received in hospital; and one patient thought the DVD was boring and preferred an interactive education on hospital TV screens or iPads. The use of technology to deliver health care messages is attracting much attention (Oosterom-Calo et al., 2014; Pfeifer Vardoulakis et al., 2012; Skeels \& Tan, 2010) and has the potential to reduce paper-based resources, encourage interactive learning and allow flexible timing of intervention delivery.

The extent of patients' understanding and subsequent behaviour change reflects that this was a pragmatic trial of a bundled intervention. Not all patients received all intervention components (Roberts et al., 2017); not all patients used all parts of the bundle; and hence behaviour change was unlikely to be consistent amongst all patients. However, some authors suggest that adherence may not require every single component of an intervention to be implemented (Carroll et al., 2007). In fact, requiring strict adherence to an intervention may undermine the working relationship between the interventionist and the patient; hence, complex interventions should be flexible and tailored to individual patients' abilities, needs and motivation (Rogers, 2010; Sidani \& Braden, 2011). Providing minimally disruptive health care interventions that minimise patient burden but are still effective can save resources, maximise adherence and improve outcomes (May, Montori, \& Mair, 2009). As the messages in the PUPCB were delivered in three ways (using a brochure, poster and DVD), patients had the opportunity to learn from or interact with the components of the intervention that they found most engaging, interesting or easy to understand. This is consistent with the philosophy of tailored, multifaceted interventions and patient-centred care.

Patients' existing knowledge, experience and intuitive routines around PU seemed to particularly affect their response to the intervention. Rogers suggests the compatibility of 
interventions with individuals’ existing values, past experiences and needs influence their uptake of innovations (Rogers, 2002). This was apparent in that patients described how they enacted the PUP messages as they were consistent with their own knowledge or instincts around repositioning, skin care and nutrition for PUP. For example, patients said they would "automatically" or "naturally" move to relieve pressure, and thought the messages "made sense”. Patients who had existing knowledge or experience with PU agreed with the PUPCB messages and felt that what they already knew was being reinforced. Similarly, others expressed PUP was not of high importance to them, and they acknowledged this was probably because they had no previous understanding or experience with PU. A study exploring inpatients' perspectives of an interactive health promotion program for heart failure found patients viewed the program favourably when it reinforced their existing knowledge and aligned with their previous experiences (Oosterom-Calo et al., 2014). Whilst other studies have reported varying extents to which compatibility influences individuals' uptake of an innovation (Emani et al., 2012; Légaré, Ratté, Gravel, \& Graham, 2008) it seemed to be an important factor in the current study.

Increased awareness around PU from the PUPCB appeared to motivate patients to enact PUP strategies. When patients learned about the consequences of PU and saw this information as relevant, useful and valuable to them, they participated actively in PUP. Rogers describes that relative advantage, 'the degree to which an innovation is perceived as better than the idea it supersedes', is a major factor influencing the uptake of innovations (Rogers, 2002). Relative advantage was one of the two most important factors perceived by patients in a study on the uptake of electronic personal health records (Emani et al., 2012). The majority of patients in the current study seemed to perceive participation in PUP as advantageous (i.e. better than doing nothing) and they described several ways in which they enacted PUP strategies. Preventative innovations are often adopted more slowly, because the relative advantage may 
be perceived as low (i.e. the reward - PUP - is not immediate) (Rogers, 2002). However, whilst a few patients did not acknowledge they were at risk of PU and tended to use more passive language when discussing PUP care (i.e. “letting” nurses enact PUP cares), nearly all patients described how they were doing at least one PUP strategy themselves. For more passive patients, these tended to be strategies that were relatively easy and seemed to have an immediate effect (i.e. moisturising skin or repositioning when uncomfortable). Yet, these simple activities may be an important reason for the PUPCB's beneficial effect.

Patients intimated their improved knowledge and understanding from the PUPCB empowered them to enact PUP strategies. Patient thought if they knew more, they could do more in regards to PUP care. Patients described a number of ways in which they self-managed their PUP care by moving/repositioning, looking after their skin and eating well; enacting what they learned from the PUPCB. Previous research indicates patients require 'appropriate, credible and sufficient knowledge in a safe and supportive environment' in order to participate in nursing care (Larsson, Sahlsten, Sjöström, Lindencrona, \& Plos, 2007). Patients perceive that 'knowledge is required to be independent, cope and manage' when participating in care (Larsson et al., 2007). Similarly in the current study, one patient explained how the knowledge she gained allowed her to independently manage and cope with a skin problem, stating "knowledge is power". One core aspect of patient participation in their care is the meaningful exchange of knowledge and information between the patient and nurse (Sahlsten, Larsson, Sjostrom, \& Plos, 2008). In this case, patients received knowledge from the PUPCB and used this to participate in, rather than being passive 'recipients' of, care. Patients who remembered and understood the intervention gave mostly active descriptions of PUP strategies (i.e. detailed how they enacted PUP) whilst patients who did not remember the intervention messages tended to use more passive language when describing PUP. 
Patients highly valued personal interactions with research staff, including the interventionists and outcome assessors. One of the most interesting findings was that the presence of the outcome assessors seemed to reinforce the intervention for patients, which was an unintended consequence of the study. Many studies have shown patients highly value human interactions in health care and this influences their satisfaction with and participation in care (Larsson et al., 2007; Lefebvre, Pelchat, Swaine, Gelinas, \& Levert, 2005; Wagner \& Bear, 2009). So, patients might have participated more in their PUP care due to the daily outcome assessor visits and these staff may have helped patients move through the 'innovation-decision' process. This unintended consequence means there may have been an interaction between the intervention and the outcome assessor visits. The control group were also visited daily by outcome assessors who performed the same assessment as on intervention patients, which may have had an effect on control patients' knowledge of PUP. However, this assessment is not likely to have had the same effect on control patients because they didn't receive the PUPCB. Hence, they were not being reminded of PUP messages by the presence of the outcome assessor (even though they were different people to the interventionists, patients knew they were part of the same trial).

\section{Limitations}

This study included 19 participants across four Australian hospitals who had participated in a PUPCB. Whilst the findings may not be generalisable to other countries, both private and public hospitals across two states were included, which increases its transferability across settings within Australia. Participants were purposively sampled and data were collected until saturation occurred, however it is possible that some views were not represented in our sample. The qualitative method used allows patients to reflect on their care (Edwards \& Titchen, 2003) and the rich data obtained enabled a broad picture of patient views to be 
expressed on a range of issues associated with the PUPCB. Another possible limitation of this study was not interviewing patients at control sites in relation to their understanding of PUP, given the unintended consequence of PUP messages being reinforced by outcome assessors among intervention patients. Interviewing control patients was not part of the process evaluation framework (Grant et al., 2013), which perhaps is an oversight or limitation to this framework.

\section{Conclusions and implications for future research}

The findings of this qualitative descriptive study of patients' responses to a PUPCB intervention are highly important to consider in relation to the interpretation of the trial's main findings and for future implementation of such interventions. Patients generally responded positively to the PUPCB and described a number of ways by which they participated in their PUP care. It seems that three simple messages that patients could understand, use, and control the extent to which they participate in, were effective in engaging patients in PUP care. An unexpected outcome was that patients perceived outcome assessors as important players in their participation in PUP, highlighting the need for reinforcement of messages and positive interactions between patients and nurses. Patients described several facilitators and barriers to participating in PUP, which should be considered if the intervention is adopted into practice. These related to the intervention content (low complexity, compatibility with existing knowledge and beliefs) and delivery (timing); and to the patients themselves (age, cognition, willingness to participate). Overall, patients found the care bundle acceptable; the main trial showed promise for clinically significant reductions in PU (Chaboyer et al., 2016); and nurses responded positively to the intervention and suggested strategies for sustaining it in practice (Roberts et al., 2016). Hence, this PUPCB may be an 
acceptable and sustainable way to promote uptake of best PUP practice and patient engagement in PUP care.

\section{Linking evidence to action}

- Educating patients can empower and motivate them to participate in PUP care

- Patients may wish to participate in their PUP care to differing extents

- Patients value human interactions in care (i.e. nurse-patient partnership)

- Educational materials and messages should be simple and easy to understand

- Interventions should align with patients' existing knowledge, values and beliefs

- Barriers to patient participation in PUP should be assessed individually 


\section{References}

Australian Bureau of Statistics. (2008). Health Literacy, Australia. Canberra: Australian Bureau of Statistics.

Australian Commission on Safety and Quality in Health Care. (2011). National safety and quality health service standards. Sydney: ACSQHC.

Braun, V., \& Clarke, V. (2006). Using thematic analysis in psychology. Qualitative Research in Psychology., 3(2), 77-101.

Carroll, C., Patterson, M., Wood, S., Booth, A., Rick, J., \& Balain, S. (2007). A conceptual framework for implementation fidelity. Implement Science, 2(1), 40.

Chaboyer, W., Bucknall, T., Webster, J., McInnes, E., Gillespie, B. M., Banks, M., . . . Tallott, M. (2016). The effect of a patient centred care bundle intervention on pressure ulcer incidence (INTACT): a cluster randomised trial. International Journal of Nursing Studies, 64, 63-71.

Craig, P., Dieppe, P., Macintyre, S., Michie, S., Nazareth, I., \& Petticrew, M. (2008). Developing and evaluating complex interventions: the new Medical Research Council guidance. International Journal of Nursing Studies, 50(5), 587-592.

Edwards, C., \& Titchen, A. (2003). Research into patients' perspectives: relevance and usefulness of phenomenological sociology. Journal of Advanced Nursing, 44(5), 450460.

Emani, S., Yamin, C. K., Peters, E., Karson, A. S., Lipsitz, S. R., Wald, J. S., . . . Bates, D. W. (2012). Patient perceptions of a personal health record: a test of the diffusion of innovation model. Journal of medical Internet research, 14(6).

European Pressure Ulcer Advisory Panel, National Pressure Ulcer Advisory Panel, \& Pan Pacific Pressure Injury Alliance. (2014). Prevention and treatment of pressure ulcers: quick reference guide. Perth, Australia: Cambridge Media.

Gillespie, B. M., Chaboyer, W., Sykes, M., O'Brien, J., \& Brandis, S. (2014). Development and pilot testing of a patient-participatory pressure ulcer prevention care bundle. Journal of Nursing Care Quality, 29(1), 74-82.

Gorecki, C., Brown, J. M., Nelson, E. A., Briggs, M., Schoonhoven, L., Dealey, C., . . . European Quality of Life Pressure Ulcer Project group. (2009). Impact of pressure ulcers on quality of life in older patients: a systematic review. Journal of the American Geriatrics Society, 57(7), 1175-1183.

Gorecki, C., Closs, S. J., Nixon, J., \& Briggs, M. (2011). Patient-reported pressure ulcer pain: a mixed-methods systematic review. Journal of pain and symptom management, 42(3), 443-459.

Grant, A., Treweek, S., Dreischulte, T., Foy, R., \& Guthrie, B. (2013). Process evaluations for cluster-randomised trials of complex interventions: a proposed framework for design and reporting. Trials, 14(1), 15.

Graves, N., \& Zheng, H. (2014). Modelling the direct health care costs of chronic wounds in Australia. Wound Practice and Research, 22(1), 20-33.

Larsson, I. E., Sahlsten, M. J., Sjöström, B., Lindencrona, C. S., \& Plos, K. A. (2007). Patient participation in nursing care from a patient perspective: a Grounded Theory study. Scandinavian Journal of Caring Sciences, 21(3), 313-320.

Latimer, S., Chaboyer, W., \& Gillespie, B. (2014). Patient participation in pressure injury prevention: giving patient's a voice. Scandinavian Journal of Caring Sciences, 28(4), 648-656. 
Lefebvre, H., Pelchat, D., Swaine, B., Gelinas, I., \& Levert, M. (2005). The experiences of individuals with a traumatic brain injury, families, physicians and health professionals regarding care provided throughout the continuum. Brain Injury, 19(8), 585-597.

Légaré, F., Ratté, S., Gravel, K., \& Graham, I. D. (2008). Barriers and facilitators to implementing shared decision-making in clinical practice: update of a systematic review of health professionals' perceptions. Patient Education and Counseling, 73(3), 526-535.

May, C., Montori, V. M., \& Mair, F. S. (2009). We need minimally disruptive medicine. British Medical Journal, 339(b2803).

Nguyen, K.-H., Chaboyer, W., \& Whitty, J. (2015). Pressure injury in Australian public hospitals: a cost of illness study. Australian Health Review, 39(3), 329-336.

Oosterom-Calo, R., Abma, T. A., Visse, M. A., Stut, W., te Velde, S. J., \& Brug, J. (2014). An interactive-technology health behavior promotion program for heart failure patients: a pilot study of experiences and needs of patients and nurses in the hospital setting. JMIR research protocols, 3(2), e32.

Pfeifer Vardoulakis, L., Karlson, A., Morris, D., Smith, G., Gatewood, J., \& Tan, D. (2012). Using mobile phones to present medical information to hospital patients. Paper presented at the Proceedings of the SIGCHI Conference on Human Factors in Computing Systems.

Queensland Government, \& Queensland Health. (2012). Metro North 2012-13 Service Agreement. Brisbane, Australia: Queensland Health.

Roberts, S., McInnes, E., Bucknall, T., Wallis, M., Banks, M., \& Chaboyer, W. (2017). Process evaluation of a cluster-randomised trial testing a pressure ulcer prevention care bundle: a mixed-methods study. Implementation Science, 12(1), 18.

Roberts, S., McInnes, E., Wallis, M., Bucknall, T., Banks, M., \& Chaboyer, W. (2016). Nurses' perceptions of a pressure ulcer prevention care bundle: a qualitative descriptive study. BMC nursing, 15(1), 64.

Rogers, E. M. (2002). Diffusion of preventive innovations. Addictive Behaviors, 27(6), 989993.

Rogers, E. M. (2010). Diffusion of innovations. New York: Simon and Schuster.

Sahlsten, M., Larsson, I., Sjostrom, B., \& Plos, K. (2008). An analysis of the concept of patient participation. Nursing Forum, 43(1), 2-11.

Sandelowski, M. (2000). Focus on research methods - whatever happened to qualitative description? Research in nursing and health, 23(4), 334-340.

Sidani, S., \& Braden, C. J. (2011). Intervention fidelity Design, evaluation, and translation of nursing interventions (pp. 125-145). West Sussex, UK: John Wiley \& Sons, Ltd.

Skeels, M., \& Tan, D. S. (2010). Identifying opportunities for inpatient-centric technology. Paper presented at the Proceedings of the 1st ACM International Health Informatics Symposium.

Vaismoradi, M., Turunen, H., \& Bondas, T. (2013). Content analysis and thematic analysis: implications for conducting a qualitative descriptive study. Nursing \& Health Sciences., 15(3), 398-405.

Wagner, D., \& Bear, M. (2009). Patient satisfaction with nursing care: a concept analysis within a nursing framework. Journal of Advanced Nursing, 65(3), 692-701.

Whiting, L. S. (2008). Semi-structured interviews: guidance for novice researchers. Nursing Standard, 22(23), 35. 